\title{
SEDENTARY BEHAVIOUR AND PHYSICAL ACTIVITY OF RANDOMISED SAMPLE OF CZECH ADULTS AGED 20-64 YEARS: IPAQ AND GPAQ STUDIES BETWEEN 2002 AND 2011
}

\author{
Dagmar Sigmundová1, Erik Sigmund', Zdeněk Hamřík ${ }^{2}$, Michal Kalmann', Jan Pavelka', Karel Frömel ${ }^{1}$ \\ 'Institute of Active Lifestyle, Faculty of Physical Culture, Palacký University Olomouc, Olomouc, Czech Republic \\ ${ }^{2}$ Department of Recreation and Leisure Studies, Faculty of Physical Culture, Palacký University Olomouc, Olomouc, Czech Republic
}

\section{SUMMARY}

Aim: The prevalence of sedentary behaviour has increased, and physical activity (PA) levels have declined with increases in urbanisation and modernisation. Researchers need to find solutions to address this lack of PA. The main aim of this study was to identify the PA level and sedentary behaviour in a randomised sample of the Czech adult population in 2002 and 2011.

Methods: To investigate PA in 20-64 year old adults, the International Physical Activity Questionnaire (IPAQ) (in 2002) and the Global Physical Activity Questionnaire (GPAQ) (in 2011) were applied. The randomised samples comprised 1,782 participants in 2002, and 1,359 participants in 2011.

Results: The results showed a significant decrease $\left(F=83.84 ; p<0.001 ; \omega^{2}=0.03\right)$ in the time spent sitting between the years 2002 and 2011. The proportion of individuals reporting low PA has increased (11.4\% in 2002 vs. $27.9 \%$ in 2011). Individuals with college/university education were more likely to have low PA levels $(\mathrm{OR}=1.51, \mathrm{p}<0.001, \mathrm{Cl}=1.21-1.68)$ compared with those with elementary or secondary education. Along with educational level, low PA levels are also associated with older age, spending more than four hours a day sitting, and the survey year.

Conclusion: Sedentary behaviour, PA, and factors influencing this behaviour may differ across countries; therefore, more trend-related research should be performed on these issues, especially in Central and Eastern Europe, where these studies are scarce.

Key words: trends, sedentary behaviour, physical activity, IPAQ, GPAQ

Address for correspondence: D. Sigmundová, Faculty of Physical Culture, Palacký University Olomouc, trída Míru 117, 77111 Olomouc, Czech Republic. E-mail: dagmar.sigmundova@upol.cz

\section{INTRODUCTION}

Adequate levels of physical activity (PA) positively influence health (1). Globalisation and urbanisation have created an environment that enhances negative changes in the population's diet and reduces PA, which results in positive energy balances (2). Overall, these changes have brought greater risks of weight gain, overweight and obesity (3). Insufficient PA is also associated with frequent occurrence of hypertension and other cardiovascular diseases, type 2 diabetes, breast cancer, bowel cancer, and death $(4,5)$. The occurrence of these non-infectious diseases is also reflected in increased health care costs (6).

To better understand PA patterns, we needed to monitor trends in the population using both longitudinal and cohort studies (7). Surveys aimed at the Czech adult population have shown that the prevalence of overweight and obesity has increased, but decreases in sedentary behaviour have not been verified $(8,9)$. The perceived decline in PA has been studied in Czech adolescents over the period of the last 10 years (10). This study identified a decrease in PA that corresponded with the fast-paced increase in the number of households with a computer and internet connection and the increase in the number of cars in the last decade (10, 11). However, only a few studies of sedentary behaviour and PA in Central and Eastern Europe have been performed $(12,13)$. Furthermore, the information about the trends in PA behaviour among the Czech population indicates that other post-communist countries in Central and Eastern Europe went through the same economic and political transition before joining the European Union. We assume that the lack of quality results and information about Central and Eastern European countries may be the reason that prevented full recognition of the importance of enhancing PA as a serious public health issue. Although several national strategic documents that support PA in Europe have been released in recent years and more member states of the European Union have prepared strategies to enhance PA, none of the Central and Eastern European states has taken either of these steps (14-16).

There are various research tools that we can use to assess PA and sedentary behaviour in different populations. The limitation of these tools is their mutual comparability. The International Physical Activity Questionnaire (IPAQ) (17) was created as a tool to allow comparisons between countries. IPAQ was originally created in 1998-2000 to provide information about PA of populations in different countries and different socio-cultural conditions and to allow international comparisons $(12,18)$. The use of the IPAQ questionnaire inspired the creation of a similarly structured equivalent version of the Global Physical Activity Questionnaire (GPAQ) $(17,19)$. GPAQ provides similar data, and results on concurrent validity showed a moderate to strong, positive relationship with the data from the IPAQ questionnaire, which had previously been validated and accepted as a suitable tool for the 
assessment of PA(17). The measurements of sedentary behaviour and physical inactivity with the items of both IPAQ and GPAQ questionnaires show good reliability (ICC $>0.77$ ), and the items of both questionnaires concerning sitting provide similar information (20). The research performed in the former Eastern bloc is unique because of its political-economic development. The countries of the former communist bloc tend to repeat the negative trends in health-related behaviour that previously took place in economically advanced Western countries (21). However, the countries in Central and Eastern Europe can learn from these negative global issues that were identified in Western Europe $(22,23)$. Due to the similar political-economic development of the former communist bloc countries, the knowledge of conditions and development of Czech inhabitants' PA behaviour can be used to create effective interventions that enhance health and PA in Central and Eastern European countries.

The main aim of the study was to describe sedentary behaviour and the PA level in a random sample of Czech adult population in 2002 and 2011. The specific goals of the study were as follows:

- to describe the differences in the time spent sitting with respect to age, gender and the year of the study;

- to describe the percentage of people who engaged in various PA levels (low, moderate, high) and the changes in PA levels between 2002 and 2011; and

- to describe the factors associated with low PA levels.

\section{MATERIALS AND METHODS}

\section{Ethics}

The current study was undertaken in the Czech Republic following an approval by the Institutional Research Ethics Committee at Faculty of Physical Culture, Palacký University in Olomouc. Participation was voluntary and no rewards or incentives for participants were offered. The participants were apprised of the aims, objectives and methods of the study before the monitoring of PA began. Confidentiality and data anonymity were carefully maintained throughout the study.

\section{Participants and Data Collection}

The examination of Czech Republic inhabitants' PA levels using the IPAQ questionnaire was carried out in the autumn of 2002. A systematic random sampling method was used to identify sampling locations from a national address database. Selected households were visited by a trained researcher following a standard protocol. All inhabitants of the household who agreed to participate and who were more than 15 years old received a questionnaire to complete. A total of 3,873 respondents participated in the study. The response rate was $58 \%$. The research sample was stratified according to region, age and gender to create a nationally representative sample. The sample for this analysis included 1,782 inhabitants of the Czech Republic aged 20-64 years.

Furthermore, the examination of Czech Republic inhabitants' PA levels using the GPAQ questionnaire was carried out in the autumn of 2011. Based on an electoral list of citizens, 1,996 respondents were randomly selected after stratification by region, age and gender (24) in order to create a nationally representative sample. Trained interviewers visited households and collected data via standardised structured interviews. The research sample consisted of 1,753 respondents aged 18-90 years. The response rate was $90 \%$. The random sample of this analysis included 1,359 Czech Republic inhabitants aged 20-64 years. The characteristics of the samples are presented in Table 1.

\section{Assessment of Sedentary Behaviour and Physical Activity}

The Czech Republic inhabitants' PA levels were assessed using the internationally standardised, short self-administered version of the IPAQ questionnaire in 2002 (18). The questionnaire contains items related to vigorous PA, moderate PA, walking and sitting. The data were processed in compliance with the IPAQ committee recommendations (25).

To assess the inhabitants' PA levels in 2011, the standardised GPAQ questionnaire was applied (19). The GPAQ questionnaire was developed by the World Health Organization (WHO) to monitor PA in different countries and contains item related to PA at work, during transportation, recreational PA, and sedentary behaviour. The obtained data were processed using the PA questionnaire analysis guide (26), and PA levels were divided into low, moderate and high.

For both questionnaires (IPAQ, GPAQ) the classification of the population into groups with low, moderate, and high PA levels is suggested $(25,26)$. A high PA level was defined when a person met any of the following criteria: vigorous activity on at least three days and achieved a minimum of 1,500 MET-minutes per week; seven days or more frequent occasions of any combination of walking or moderate or vigorous activity that achieved a

Table 1. Population characteristics by survey year

\begin{tabular}{|c|c|c|}
\hline & 2002 & 2011 \\
\hline$n$ & 1,782 & 1,359 \\
\hline \multicolumn{3}{|l|}{ Age $(\%)$} \\
\hline 20-34 years & 36.5 & 35.0 \\
\hline $35-49$ years & 35.1 & 33.9 \\
\hline 50-64 years & 28.4 & 31.1 \\
\hline \multicolumn{3}{|l|}{ Gender (\%) } \\
\hline Male & 50.7 & 50.3 \\
\hline Female & 49.3 & 49.7 \\
\hline \multicolumn{3}{|l|}{ Education (\%) } \\
\hline Elementary & 4.1 & 6.0 \\
\hline Secondary & 74.6 & 75.5 \\
\hline University & 21.3 & 18.5 \\
\hline \multicolumn{3}{|c|}{ Physical activity level (\%) } \\
\hline Low & 11.4 & 27.9 \\
\hline Moderate & 31.0 & 21.4 \\
\hline High & 57.6 & 50.7 \\
\hline \multicolumn{3}{|c|}{ Sedentary behaviour (\%) } \\
\hline$<4$ hour per day & 19.4 & 33.8 \\
\hline$\geq 4$ hour per day & 80.6 & 66.2 \\
\hline
\end{tabular}


minimum of 3,000 MET-minutes per week. A moderate PA level was defined when a person did not meet the criteria for the "high" category but met any of the following criteria: three or more days of vigorous activity for at least 20 minutes per day; five or more days of moderate-intensity activity or walking for at least 30 minutes per day; or five and more days of any combination of walking, moderate or vigorous intensity activity that achieved a minimum of 600 MET-minutes per week. A low PA level was defined when a person did not meet any of the aforementioned criteria $(25,26)$.

\section{Statistical Analyses}

Statistical analyses were performed using STATISTICA v. 10 and IBM's SPSS v. 19. To assess the differences between the proportions of PA classifications (low, moderate or high), Pearson's $\chi^{2}$ test and analyses of adjusted residuals were applied. Logistic regression was used to estimate the probability of being categorised as having a low PA level. The dependent variable was the category of low PA, and the independent variables were the year of the study $(2002,2011)$, gender, age category (20-34, 35-49 and 50-64 years), and time spent sitting ( $<4$ hour per day, $\geq 4$ hour per day). MANOVA and ANOVA of the main effects were applied for more detailed analyses of the time spent sitting as the independent variable and the age, gender and the year of the study as the dependent variables (factors). An alpha level of 0.05 was used to evaluate statistical significance, and the effect size coefficient $\left(\omega^{2}\right)$ was also used (27).

\section{RESULTS}

\section{Sedentary Behaviour}

Irrespective of the participants' age, the average time spent sitting in 2002 was 6.25 hours per day in men and 6.66 hours per day in women, whereas in 2011 men spent sitting on average 5.42 hours per day and women 5.48 hours per day.

Regarding the sedentary time (Fig. 1), significant differences between 2002 and $2011\left(\mathrm{~F}=83.84 ; \mathrm{p}<0.001 ; \omega^{2}=0.03\right)$ and significant differences between men and women $(\mathrm{F}=5.74 ; \mathrm{p}=0.017$; $\left.\omega^{2}<0.01\right)$ were confirmed. However, the differences between

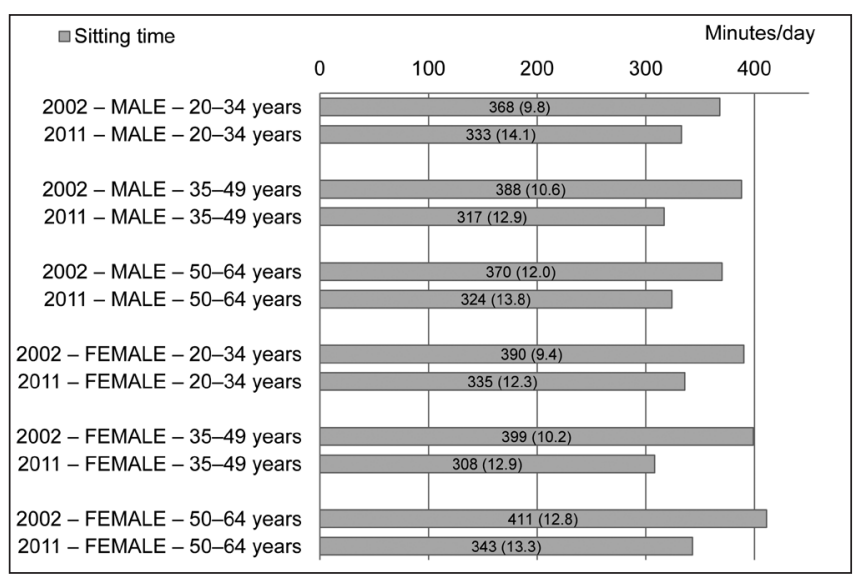

Fig. 1. Mean (standard error) sitting time per day according to the year of survey, gender and age category. the age groups were not significant for this variable $(\mathrm{F}=1.36$; $\left.\mathrm{p}=0.058 ; \omega^{2}<0.01\right)$. An analysis of variance revealed a main effect indicating that adult participants in 2002 spent significantly more time sitting than in 2011. Females spent more time sitting than males. Based on the MANOVA model, the interactions between age, gender, year of the study, and sedentary time were not significant.

\section{Physical Activity Levels - in 2002 and 2011}

Out of the total of 1,782 participants in 2002 and 1,359 participants in 2011, in both surveys more than half of adults reported a high PA level (Table 1). Low PA levels were reported in $11.4 \%$ of adults in 2002 and $27.9 \%$ of adults in 2011 .

A detailed comparison of 2002 and 2011 based on Pearson's $\chi^{2}$ test revealed that the proportions of participants in the categories of low, moderate and high PA levels significantly $(p<0.01)$ changed among both males and females in all age groups. In all age categories (20-34, 35-49 and 50-64 years), the results of an adjusted residual analysis showed a significant increase $(\mathrm{p}<0.05)$ in the proportions of males and females in the low PA category. Furthermore, with the exception of females aged 50-64 years, the proportion of adults in the moderate PA category showed a significant decline. The high PA category showed no significant changes between 2002 and 2011 in the age categories below 50 years of age; however, the age group of 50-64 years exhibited a significant decline $(\mathrm{p}<0.05)$ in the proportions of both males and females in the high PA level category.

\section{Factors Associated with Low Physical Activity Levels}

The results of logistic regression (Table 2) revealed that adult participants in the 2011 study were approximately 3.6 times more likely to be classified in the low PA category than the participants in the 2002 study. Similarly, adults in the age categories of 35-49 and 50-64 years were more likely to fall into the low PA level category than were young adults aged 20-34 years. Universityeducated adults (in 2011) and also participants who spent more than four hours a day sitting were more likely to be in the low PA level category. In contrast, gender was not significantly associated with low PA levels, and the interactions between the dependent variables and the year of the study were not significant. In summary, factors such as the 2011 survey year, older age, and spending more than four hours a day sitting were associated with higher odds of having a low PA level.

\section{DISCUSSION}

The aim of this study was to identify the changes in sedentary behaviour and PA in a randomized sample of the Czech adult population between 2002 and 2011. Although we might expect that significant declines in PA would be accompanied by increases in sedentary behaviour (28), the results of this study are not that clear regarding this issue.

The sedentary time decreased from 2002 to 2011, but other studies have shown increases in sedentary behaviour (29). Despite the observed decline in sedentary time in Czech adults, respondents in 2011 spent, on average, more than 5.4 hours per day 


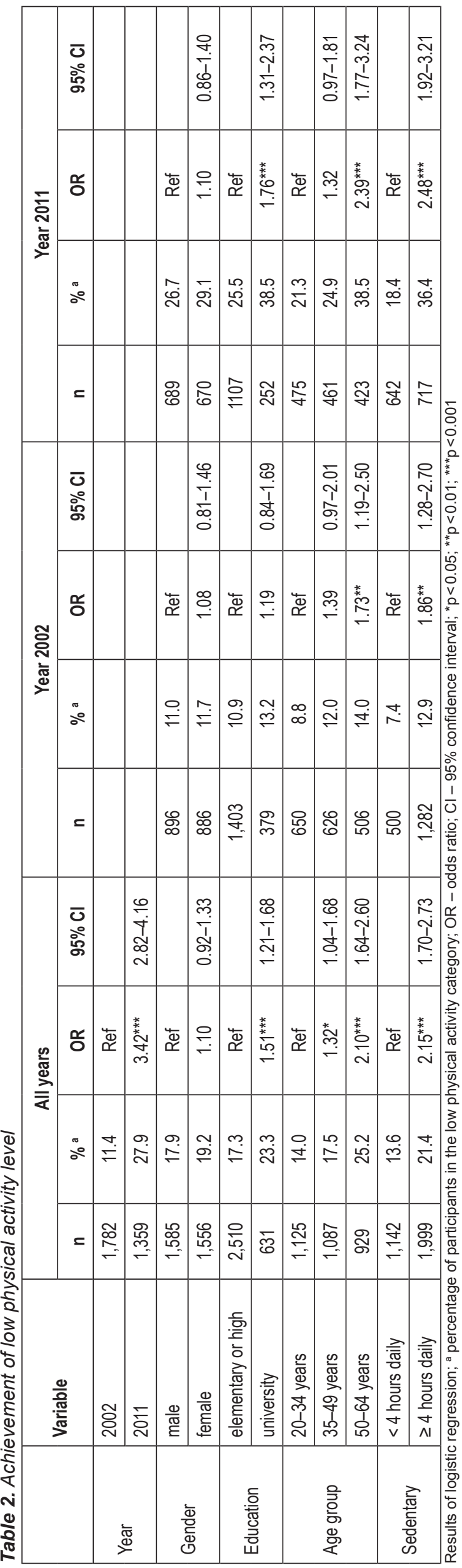

being sedentary. Sedentary behaviour is associated with cardiometabolic and other risk factors $(4,5)$. The effects of sedentary behaviour in adults are specific to various types of sedentary behaviour, therefore, we can assume that this scientific area is complicated and cannot be understood simply as an absence of PA (30). Moreover, results indicated that sedentary behaviour can induce various risk factors that are independent of PA level (25).

PA is relatively independent of sedentary behaviour, which means that these two behaviours do not directly displace each other in youth and adults $(31,32)$. PA is an important determinant of health, and adequate PA has preventive qualities. Low PA levels represent a risk factor for diseases such as high blood pressure, other cardiovascular diseases and diabetes $(4,5)$. The results of this study confirmed an increase in the number of adults with low PA levels from 2002 to 2011. Similar trends were identified in a cross-sectional study of the Argentine National Risk Factor Surveys. This study reported that the prevalence of low PA levels and obesity increased in the period from 2005 to 2009 (33). However, an opposite trend was also found, when the percentage of inactive Danish adults was decreased between 2000 and 2010 (34). Our study of the Czech adult population showed that older people and people who spent more time sitting had higher risks of being classified in the low PA category. The Survey of Health Ageing and Retirement in Europe showed that older people, low PA levels and declines in PA are associated with declines in selfevaluations of health (35).

A positive finding of the present study is that more than a half of adult individuals achieve a high PA level. A comparison of results of the IPAQ short and GPAQ questionnaires revealed that compared with 2002, 2011 saw an increase in the proportion of men and women classified in the low PA-level category. Although the IPAQ and GPAQ questionnaires are comparable, they are structurally different. The short form of IPAQ contains fewer questions than GPAQ (36). However, the longest version of the questionnaire is the IPAQ long. Some studies point to the fact that the IPAQ short overestimates inactivity and systematically underestimates PA levels compared with the long version of IPAQ $(37,38)$. In assessing PA using IPAQ and GPAQ in small samples, the decisive factor can be the stability of PA behaviour, where the IPAQ and GPAQ questionnaires report a higher degree of association in individuals with a stable behavioural pattern. The IPAQ short and GPAQ report good reliability and validity at a population level as well as they have mutual comparability (19, $20,39)$. Even if we took into consideration studies in which the IPAQ short overestimates physical inactivity and underestimates PA levels, in the assessment of changes in the IPAQ short in 2002 and GPAQ in 2011, we could expect even higher increase in the proportion of individuals in the moderate and high PA-level category. Unfortunately, no study at a population level has been published that would compare the results of PA according to the IPAQ short and GPAQ. Therefore, these results should be interpreted with a degree of caution.

Contrary to the results concerning Czech adults, the results revealed that the PA levels in Polish adults were higher at the beginning of 21 st century than those in the 1990s (12). Swiss adults also reported significant increase in physical activity from 1999 to 2009 (40). High PA levels are associated with walking and cycling, and both males and females with high PA levels have healthy BMIs (41). In contrast, only approximately 19\% 
of Czech adults included in our study were in both surveys in the low PA level category, which, unlike the high PA level, is associated with an elevated occurrence of being overweight and obese (41). A previous study of the Czech population revealed increases in the proportions of people who are overweight and obese; however, a decline in PA was not confirmed (8). Nevertheless, more than $60 \%$ of adults are assessed as being sedentary (13). In Poland, low and inadequate PA levels were confirmed in two-thirds of the adult population; however, this research did not examine the differences between PA in Polish people and other Europeans (12).

Furthermore, the results of our study showed that people with a university education were more likely to be in the low PA level category than people with lesser education. This finding corresponds with findings obtained using the IPAQ short questionnaire in the Czech population in 2008 (42). In contrast, in the French middle age population, women with higher education met the health recommendations for PA more frequently than women with lesser education (43). The influence of education on PA differs in various countries. For example, a Norwegian cross-sectional study showed that interventions aimed at changing the psychological correlates of PA can be effective regardless of gender, weight and education (44).

As expected, our study showed that older and predominantly sedentary people had higher probabilities of being in the low PA-level category. In contrast, older French adults were more likely to meet the PA recommendations (43). An international comparison of 20 countries argued that PA is inversely associated with sedentary behaviour. Participants who report low activity in IPAQ are three times more likely to be in the top quintile for time spent sitting compared to those who report high PA levels (45).

Despite the fact that both studies were carried out in representative samples of the Czech population using valid and internationally standardised tools for assessing PA (IPAQ, GPAQ), this study has limitations. The validation of the GPAQ questionnaire for Central European countries has not yet been published, though it is shortcoming. Another general limitation is the fact that the data in questionnaire surveys are biased with subjective perceptions. We also do not have information about how many individuals are relatives. Analyses related to education and low PA level may be influenced by the fact that adults aged 20 years may not have definitely completed education, and this bias could affect the result in this analysis.

In summary, a positive finding arising from the present study is a decrease in the time spent sitting, although adults still spend over 5.4 hours a day sitting, on average. This study also shows that the proportion of adults in the moderate PA-level category is decreasing and that the proportion of people in the low PAlevel category is increasing. Contrary to our expectations, the Czech population with college/university education exhibited a greater probability of being in the low PA-level category compared with people with secondary or elementary education. Older people and those who are sedentary for more than four hours a day showed increased probabilities of being in the low PA-level category. Together, the results of this study and other studies imply that PA and sedentary behaviours can differ across countries. Therefore, more research on the trends of sedentary behaviour and PA should be performed, especially in Central and Eastern European countries.

\section{Acknowledgements}

This work was supported by the research grant of the Czech Science Foundation Reg. No. GA14-02804S and by the Czech Ministry of Education, Youth and Sports under Contract No. LG 14043. Specifically, the authors would like to thank Dr Petr Sadílek, the INRES agency, and the Centre for Kinanthropology Research, who collected data for this study.

\section{Conflict of Interest}

None declared

\section{REFERENCES}

1. Baceviciene M, Luksiene DI, Cesnaitiene VJ, Raubaite S, Peasey A Tamosiunas A. Dose-response association between physical activity and metabolic syndrome. Cent Eur J Med. 2013;8(2):273-82.

2. Malik VS, Willett WC, Hu FB. Global obesity: trends, risk factors and policy implications. Nat Rev Endocrinol. 2013;9(1):13-27.

3. Cunha DB, de Almeida RMVR, Sichieri R, Pereira RA. Association of dietary patterns with BMI and waist circumference in a low-income neighbourhood in Brazil. Br J Nutr. 2010;104(6):908-13.

4. Lee IM, Shiroma EJ, Lobelo F, Puska P, Blair SN, Katzmarzyk PT Effect of physical inactivity on major non-communicable diseases worldwide: an analysis of burden of disease and life expectancy. Lancet. 2012;380(9838):219-29.

5. Simoes EJ, Mariotti S, Rossi A, Heim A, Lobello F, Mokdad AH, et al. The Italian health surveillance (SiVeAS) prioritization approach to reduce chronic disease risk factors. Int J Public Health. 2012;57(4):719-33.

6. Janssen I. Health care costs of physical inactivity in Canadian adults. Appl Physiol Nutr Metab. 2012;37(4):803-6.

7. Duncan MJ, Vandelanotte C, Caperchione C, Hanley C, Mummery WK. Temporal trends in and relationships between screen time, physical activity, overweight and obesity. BMC Public Health. 2012;12(1):1060.

8. Sigmundová D, Sigmund E, Frömel K, Suchomel A. Gender differences in physical activity, sedentary behavior and BMI in the Liberec region: the IPAQ study in 2002-2009. J Hum Kinet. 2011 Jun;28:123-31.

9. Cífková R, Škodová Z, Bruthans J, Adámková V, Jozífová M, Galovcová $\mathrm{M}$, et al. Longitudinal trends in major cardiovascular risk factors in the Czech population between 1985 and 2007/8. Czech MONICA and Czech post-MONICA. Atherosclerosis. 2010;211(2):676-81.

10. Sigmundová D, El Ansari W, Sigmund E, Frömel K. Secular trends: a ten-year comparison of the amount and type of physical activity and inactivity of random samples of adolescents in the Czech Republic. BMC Public Health. 2011 Sep 26;11:731

11. Czech Statistical Office. Statistical yearbook of the Czech Republic 2010. 1. vyd. Praha: Scientia; 2010.

12. Piątkowska M. Self-rated physical activity level across Europe - Poland and other European countries. Biol Sport. 2012;29(1):23-31.

13. Hamrik Z, Sigmundová D, Kalman M, Pavelka J, Sigmund E. Physical activity and sedentary behaviour in Czech adults: results from the GPAQ study. Eur J Sport Sci. 2014;14(2):193-8.

14. Daugbjerg SB, Kahlmeier S, Racioppi F, Martin-Diener E, Martin B Oja P, et al. Promotion of physical activity in the European region: content analysis of 27 national policy documents. J Phys Act Health. 2009;6(6):805-17.

15. Oja P, Bull FC, Fogelholm M, Martin BW. Physical activity recommendations for health: what should Europe do? BMC Public Health. 2010 Jan $11 ; 10: 10$.

16. Kalman M, Hamrik Z. Is the low level of physical activity a public policy issue? Tělesná kultura. 2013;36(2):96-114. (In Czech.)

17. Bull FC, Maslin TS, Armstrong T. Global physical activity questionnaire (GPAQ): nine country reliability and validity study. J Phys Act Health. 2009;6(6):790-804.

18. Craig CL, Marshall AL, Sjöström M, Bauman AE, Booth ML, Ainsworth $\mathrm{BE}$, et al. International physical activity questionnaire: 12-country reliability and validity. Med Sci Sports Exerc. 2003;35(8):1381-95.

19. Armstrong T, Bull F. Development of the World Health Organization Global Physical Activity Questionnaire (GPAQ). J Public Health. 2006;14(2):66-70. 
20. Fonseca AA, Lemos DMC, Arenas MO. Reproducibilidad del tiempo en posición sedente evaluado con el International Physical Activity Questionnaire (IPAQ) y el Global Physical Activity Questionnaire (GPAQ). MedUNAB. 2010;13(1):5-12.(In Spanish.)

21. Branca F, Nikogosian H, Lobstein T, editors. The challenge of obesity in the WHO European Region and the strategies for response: summary. Copenhagen: WHO Regional Office for Europe; 2007.

22. Knai C, Suhrcke M, Lobstein T. Obesity in Eastern Europe: an overview of its health and economic implications. Econ Hum Biol. 2007;5(3):392408.

23. Melin R, Fugl-Meyer KS, Fugl-Meyer AR. Life satisfaction in 18-to 64-year-old Swedes: in relation to education, employment situation, health and physical activity. J Rehabil Med. 2003;35(2):84-90.

24. Czech Statistical Office. Statistical yearbook of the Czech Republic 2012. 1. vyd. Praha : Český statistický úr̆ad, 2012.

25. Thorp AA, Owen N, Neuhaus M, Dunstan DW. Sedentary behaviors and subsequent health outcomes in adults: a systematic review of longitudinal studies, 1996-2011. Am J Prev Med. 2011;41(2):207-15.

26. World Health Organization. Global physical activity questionnaire (GPAQ): analysis guide. Geneva: WHO; 2012.

27. Cortina JM, Nouri H. Effect size for ANOVA design. Thousand Oaks, CA: Sage Publications; 2000.

28. Ng SW, Popkin BM. Time use and physical activity: a shift away from movement across the globe. Obes Rev. 2012;13(8):659-80.

29. Proper KI, Singh AS, van Mechelen W, Chinapaw MJ. Sedentary behaviors and health outcomes among adults: a systematic review of prospective studies. Am J Prev Med. 2011;40(2):174-82.

30. Rhodes RE, Mark RS, Temmel CP. Adult sedentary behavior: a systematic review. Am J Prev Med. 2012;42(3):e3-28.

31. Pearson N, Braithwaite RE, Biddle SJ, van Sluijs EM, Atkin AJ. Associations between sedentary behaviour and physical activity in children and adolescents: a meta-analysis. Obes Rev. 2014;15(8):666-75.

32. Schmid D, Ricci C, Leitzmann MF. Associations of objectively assessed physical activity and sedentary time with all-cause mortality in US adults: the NHANES study. PloS One. 2015;10(3):e0119591.

33. Linetzky B, De Maio F, Ferrante D, Konfino J, Boissonnet C. Sexstratified socio-economic gradients in physical inactivity, obesity, and diabetes: evidence of short-term changes in Argentina. Int J Public Health. 2013;58(2):277-84.

34. Molsted S, Johnsen NF, Snorgaard O. Trends in leisure time physical activity, smoking, body mass index and alcohol consumption in Danish adults with and without diabetes: a repeat cross-sectional national survey covering the years 2000 to 2010. Diabetes Res Clin Pract. 2014;105(2):217-22.
35. Verropoulou G. Determinants of change in self-rated health among older adults in Europe: a longitudinal perspective based on SHARE data. Eur J Ageing. 2012;9(4):305-18.

36. Bauman A, Phongsavan P, Schoeppe S, Owen N. Physical activity measurement--a primer for health promotion. Promot Educ. 2006;13(2):92103.

37. Hallal PC, Victora CG. Reliability and validity of the International Physical Activity Questionnaire (IPAQ). Med Sci Sports Exerc. 2004;36(3):556.

38. Sigmund E, Sigmundová D, Mitáš J, Chmelík F, Vašíčková J, Frömel $\mathrm{K}$. Variability of selected indicators of physical activity in a randomized sample of the Czech population between the years 2003-2006: results from the short and long self-administered format of the IPAQ questionnaire. Acta Univ Palacki Olomuc Gymn. 2009;39(2):23-31.

39. Araujo TL, Matsudo SM, Matsudo VK. Comparison of physical activity level using two international physical activity questionnaires: GPAQ and IPAQ. Med Sci Sports Exerc. 2005;37(5 Suppl):S111.

40. Guessous I, Gaspoz J-M, Theler JM, Kayser B. Eleven-year physical activity trends in a Swiss urban area. Prev Med. 2014 Feb;59:25-30.

41. Belohlavek R, Sigmund E, Zacpal J. Evaluation of IPAQ questionnaires supported by formal concept analysis. Inform Sciences. 2011;181(3):1774-86.

42. Vašíčková J, Roberson D, Frömel K. The education level and sociodemographic determinants of physical activity in Czech adults. Hum Mov. 2012;13(1):54-64.

43. Bertrais S, Preziosi P, Mennen L, Galan P, Hercberg S, Oppert JM. Sociodemographic and geographic correlates of meeting current recommendations for physical activity in middle-aged French adults: the Supplémentation en Vitamines et Minéraux Antioxydants (SUVIMAX) Study. Am J Public Health. 2004;94(9):1560-6.

44. Hansen BH, Ommundsen Y, Holme I, Kolle E, Anderssen SA. Correlates of objectively measured physical activity in adults and older people: a cross-sectional study of population-based sample of adults and older people living in Norway. Int J Public Health. 2014;59(2):221-30.

45. Bauman A, Ainsworth BE, Sallis JF, Hagströmer M, Craig CL, Bull FC, et al. The descriptive epidemiology of sitting. A 20-country comparison using the International Physical Activity Questionnaire (IPAQ). Am J Prev Med. 2011;41(2):228-35.

Received October 23, 2014 Accepted in revised form September 17, 2015 